of congenital syphilis has increased by $23 \%$ since 2005 and, in 2008, 141 infants were infected with HIV, despite the fact that the CDC recommends routine prenatal syphilis and HIV screening. To encourage compliance with guidelines, many payers rely on claims data to track utilisation. In this study, the percentage of women screened for syphilis or HIV during their pregnancy was estimated using MarketScan claims data, as a measure of compliance and of the feasibility of using these data for assessment of prenatal screening. Methods We analysed data from the MarketScan database, that allows linkage of de-identified patient and utilisation data from varying sites of care. The analysis was restricted to women who delivered a live birth in 2008, and who were continuously enrolled in one health plan for at least 293 days prior to delivery. The eligible population was identified using the coding algorithm for a live birth defined by HEDIS.

Results Among the 220571 women with a live birth in 2008, 87.0\% received a syphilis test during their pregnancy, $74.1 \%$ received an HIV test and $73.3 \%$ received both. The test rate for syphilis was highest for those 31-35 yrs (88.2\%) and lowest for those 15-20 yrs (78.3\%). The test rate for HIV was highest among those $21-25$ yrs $(75.2 \%)$ and lowest among those $15-20$ yrs (70\%). Both prenatal syphilis and HIV test rates were highest among those insured by a preferred provider plan. Regionally, testing rates were highest in the South $(89.8 \%$ for syphilis and $82.1 \%$ for HIV) and lowest in the Northeast for syphilis (82.8\%) and in the West for HIV (62.9\%).

Conclusions The results suggest fairly good compliance with guidelines for prenatal HIV and syphilis screening, although utilisation still varies by age, insurance type and geographic region. The MarketScan database represents individuals covered by employment-based health plans, which is how most Americans are insured. These data provide a unique opportunity to assess utilisation among the insured, at a time when the USA is poised to move a larger proportion of the population into this group. Tracking of yearto-year changes could help public health and healthcare organizations partner to improve care and target interventions to ensure equal access to these critical services.

\section{P1-S6.21 CHLAMYDIA TRACHOMATIS SCREENING INITIATIVE AMONG FEMALE US ARMY SOLDIERS DEPLOYED TO KOREA}

doi:10.1136/sextrans-2011-050108.245

\begin{abstract}
${ }^{1} \mathrm{~N}$ Jordan, ${ }^{1} \mathrm{~N}$ Clemmons, ${ }^{2} \mathrm{~J}$ Gaydos, ${ }^{3} \mathrm{~J}$ Fishman, ${ }^{3} \mathrm{~T}$ Jacobsmuhlen, ${ }^{3} \mathrm{H}$ Choon Lee, ${ }^{3} \mathrm{M}$ Meyers, ${ }^{3} \mathrm{~T}$ Klein. ${ }^{1}$ US Army Public Health Command, Aberdeen Proving Grounds, Edgewood, USA; ${ }^{2}$ Armed Forces Health Surveillance Center, Silver Spring, USA; ${ }^{3}$ USA MEDDAC, Republic of South Korea
\end{abstract}

Objectives To determine the prevalence of Chlamydia trachomatis (Ct) infections among female US Army soldiers deployed to the Republic of Korea; to identify high-risk groups; and to develop data to inform Army leaders and public health decision makers regarding the program's impact.

Methods All females reporting (in-processing) to the Eighth US Army, Republic of Korea, between 1 November 2007 and 31 December 2009 received an educational STI brief and a questionnaire, and were requested to provide a urine sample for $\mathrm{Ct}$ and gonorrhoea testing using the Aptima Combo 2 Assay. Contact tracing was conducted for all identified cases. Descriptive analyses of the population of interest and data collected during calendar years 2008-2009 were performed. A logistic regression model was generated to identify statistically significant risk factors.

Results Women deployed to Korea differed somewhat demographically when compared to the overall female Army population; a higher proportion of known high risk groups was observed (eg, women under 25 years of age). A total of 3761 women were screened during the 2 calendar years, with an overall positive rate of $5.7 \%$. Ct rates were as high as $11.4 \%$ among women under 20 years, and decreased with increasing age. Rates varied considerably by race, with the highest rates observed among American Indians/Alaskan natives $(9.3 \%)$, followed by Black females (6.5\%). The Eighth US Army screening program was associated with diagnosis of infections at an earlier age (22.1 years on average during the screening program, as compared to 26.1 years on average before the program was initiated). Statistically significant differences were observed, with higher rates found among Black women and women $<25$ years of age.

Conclusion A high prevalence of infection was observed, highlighting the need for a continued Eighth US Army screening program. Furthermore, the program demonstrated that universal screening during Army in-processing was feasible and resulted in detection of $\mathrm{Ct}$ at earlier ages, which likely reduces disease transmission, medical complications, and associated costs with treatment. These findings have implications not only for the continuation of the program in the Republic of Korea, but also for the expansion of such programs during in-processing activities among other military high risk populations (eg, Army recruits)

\section{P1-S6.22 CHARACTERISTICS OF CHLAMYDIA TESTING BY A LARGE COMMERCIAL LABORATORY CORPORATION, USA, 2008-2010}

doi:10.1136/sextrans-2011-050108.246

${ }^{1} \mathrm{C}$ Kent, ${ }^{1} \mathrm{G}$ Tao, ${ }^{1} \mathrm{~K}$ Hoover, ${ }^{2} \mathrm{~B}$ Body, ${ }^{2} \mathrm{M}$ Nye. ${ }^{1} \mathrm{CDC}$, Atlanta, USA; ${ }^{2}$ Laboratory Corporation of America, USA

Background US guidelines recommend chlamydia screening for all sexually active young women, HIV-infected persons, and men who have sex with men (MSM). Several types of chlamydia tests are available, including nucleic amplification tests (NAATs), DNA probe, and culture. Different assays have different sensitivity and specificity, and sensitivity and specificity also varies by specimen type. Because reported chlamydia trends could be impacted by shifts in the proportion of tests and specimen types used by providers and laboratories, it is important to monitor changes in test usage to help interpret reported trends in morbidity. We examined chlamydia testing data from one of the two largest commercial laboratories in the USA

Methods Among chlamydia tests performed from June 2008-to July 2010 by this laboratory, we assessed the frequency of test types used by year, sex, specimen type, and positivity of these tests.

Results During these 2 years, 2.9 million specimens were tested for chlamydia. Among those tested, $86 \%$ were women. Tests performed were: $77 \%$ NAATs, $23 \%$ DNA probes, and $0.3 \%$ cultures. Between year 1 and 2, NAATs increased from 73\% to $80 \%$ of all tests, and DNA probes decreased from $27 \%$ to $19 \%$. Test type varied by sex; $88 \%$ of tests among men were NAATs compared to $75 \%$ among women. The top specimen types among women tested by NAAT were: $45 \%$ cervical, $35 \%$ urine and $18 \%$ vaginal. Chlamydia positivity varied by test type: $5.1 \%$ NAAT, $1.4 \%$ DNA probe, and $2.3 \%$ culture.

Conclusions NAATs were the most frequently used chlamydia test and their use increased over the evaluation period, creating a smaller market for other tests. Although vaginal swabs are the most sensitive specimen for testing women by NAAT, currently they are the least common specimen type used for testing. This may reflect the delayed introduction (FDA clearance) of the vaginal swab compared to other collection devices. Stocking the wide assortment of collection devices required for testing different specimens in different populations might also be a barrier for providers. Because 